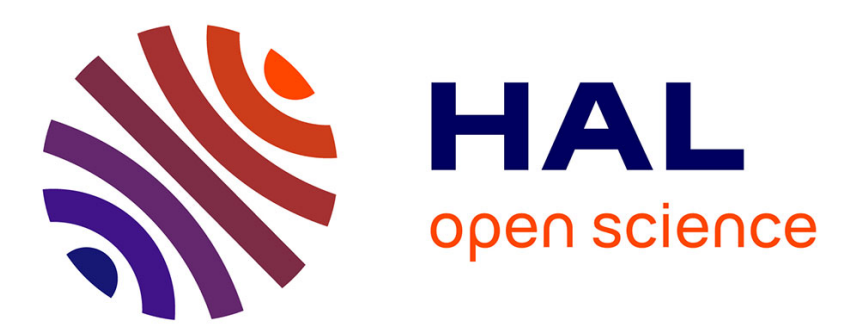

\title{
Etude expérimentale de différents modes de conduction par sauts dans un alliage désordonné $\mathrm{Nb}-\mathrm{N}$
}

Jérome Chevalier, J. Baixeras, P. Andro

\section{To cite this version:}

Jérome Chevalier, J. Baixeras, P. Andro. Etude expérimentale de différents modes de conduction par sauts dans un alliage désordonné Nb-N. Journal de Physique, 1981, 42 (3), pp.405-411. 10.1051/jphys:01981004203040500 . jpa-00209024

\section{HAL Id: jpa-00209024 https://hal.science/jpa-00209024}

Submitted on 1 Jan 1981

HAL is a multi-disciplinary open access archive for the deposit and dissemination of scientific research documents, whether they are published or not. The documents may come from teaching and research institutions in France or abroad, or from public or private research centers.
L'archive ouverte pluridisciplinaire HAL, est destinée au dépôt et à la diffusion de documents scientifiques de niveau recherche, publiés ou non, émanant des établissements d'enseignement et de recherche français ou étrangers, des laboratoires publics ou privés. 


\title{
Etude expérimentale de différents modes de conduction par sauts dans un alliage désordonné $\mathbf{N b}-\mathbf{N}\left({ }^{*}\right)$
}

\author{
J. Chevalier, J. Baixeras et P. Andro \\ Laboratoire de Génie Electrique des Universités Paris VI et Paris XI (**), \\ Ecole Supérieure d'Electricité, Plateau du Moulon, 91190 Gif-sur-Yvette, France
}

(Reçu le 9 juin 1980, accepté le $1^{\mathrm{er}}$ décembre 1980)

\begin{abstract}
Résumé. - Nous présentons dans cet article des résultats expérimentaux de variation de la résistance en fonction de la température d'alliages $\mathrm{Nb}-\mathrm{N}$ non métalliques désordonnés. Le comportement observé permet de distinguer trois régimes bien distincts et met en particulier en évidence un écart net à la loi de Mott $\left(\sigma=\sigma_{0} \exp \left[\left(T / T_{0}\right)^{-1 / 4}\right]\right)$ aux plus basses températures; la loi expérimentale observée dans ce cas peut s'expliquer en faisant intervenir les corrélations électroniques. Nous avons montré que la prise en compte de celles-ci, par le modèle de Efros et Shklowskii par exemple, permet de bien rendre compte des résultats expérimentaux et d'obtenir des valeurs raisonnables pour la densité d'états au niveau de Fermi et pour la longueur de localisation des électrons dans le gap de mobilité.

Abstract. - In this paper we present experimental results concerning the variation of the resistance $v s$. temperature for non-metallic disordered alloys of $\mathrm{Nb}-\mathrm{N}$. The observed behaviour clearly exhibits three different regimes ; in particular Mott's law is no longer obeyed at the lowest temperatures; the experimental variation in this case can be explained by electronic correlations. We have taken these correlations into account through the model of Efros and Shklowskii and therefore we have obtained : i) a good agreement with the experimental results; ii) reasonable values for the density of states at the Fermi level and for the length of localization of the electrons in the mobility gap.
\end{abstract}

1. Introduction. - Le désordre dans les solides cristallins se traduit en particulier par l'apparition d'états électroniques localisés. Ces états sont caractérisés d'une part par une fonction d'onde, dont l'amplitude décroît très rapidement à partir d'un site géographique bien déterminé, et d'autre part par une mobilité extrêmement faible en comparaison de celle des états étendus des bandes de conduction ou de valence. Dans le cas des semiconducteurs ces états se situent dans ce qui était la bande interdite du cristal; lorsque la densité de ces états est importante, le mode de conduction diffère profondément de celui qui est observé dans les semiconducteurs cristallins, le transport électronique étant assuré par des sauts thermiquement activés d'un état localisé à un autre. En étudiant ce phénomène Mott [1] a prévu théoriquement l'existence de deux régimes de conduction suivant la valeur de la température :

(*) Ce travail fait partie de la thèse de Docteur de Spécialité soutenue par J. Chevalier devant l'Université Paris VI.

(**) Associé au C.N.R.S. L.A., $\mathrm{n}^{\circ} 127$ i) Dans la gamme des hautes températures les sauts ont lieu entre proches voisins et l'expression de la conductivité en fonction de la température peut se mettre sous la forme

$$
\sigma(T)=\sigma_{01} \exp \left(\frac{\Delta E}{k_{\mathbf{B}} T}\right)
$$

$\Delta E$ étant l'écart énergétique moyen entre proches voisins et $\sigma_{01}$ une constante dépendant de la nature du matériau.

ii) Pour les basses températures si $k_{\mathrm{B}} T$ est insuffisant pour permettre à un électron localisé d'accéder à un état localisé proche voisin, cet électron ne peut trouver d'état énergétique accessible que dans des états localisés plus éloignés.

L'analyse de ce dernier type de conduction thermiquement activée par sauts à distance variable conduit à l'expression de la conductivité nommée loi de Mott :

$$
\sigma=\sigma_{04} \mathrm{e}^{-\left(T / T_{0(4))^{-1 / 4}}\right.} .
$$


La température $T_{0(4)}$ est donnée par l'expression :

$$
T_{0(4)}=\frac{18,1}{k_{\mathrm{B}} d^{3} N\left(E_{\mathrm{F}}\right)}
$$

dans laquelle $N\left(E_{\mathrm{F}}\right)$ est la densité d'états localisés supposée constante dans le gap de mobilité, $d$ représente l'extension de la fonction d'onde de l'électron localisé ;

$$
\sigma_{0(4)}=e^{2} N\left(E_{\mathrm{F}}\right) R^{2} v
$$

où $v$ est une fréquence typique de phonons, et $R$ est la distance de saut la plus probable, calculée par maximisation de la probabilité de saut :

$$
R=\left[\frac{9 d}{8 N\left(E_{\mathrm{F}}\right) k_{\mathrm{B}} T}\right]^{1 / 4} .
$$

Les paramètres $\sigma_{0(4)}$ et $T_{0(4)}$ ont été reliés aux propriétés microscopiques du matériau d'abord par Mott et ensuite par Ambegaokar, Halperin et Langer [2].

Le type de variation de la conductivité prévu par Mott est conforme aux résultats expérimentaux de nombreux auteurs dans le domaine des basses températures. Toutefois, certains d'entre eux signalent un régime en

$$
\sigma=\sigma_{0(x)} \mathrm{e}^{-\left(T / T_{0(x)}\right)^{-1 / x}}
$$

avec $x=2$ ou 3 ; dans certains cas, $x$ passe de 4 à 2 lorsque la température s'abaisse. Pour expliquer les diverses valeurs de $x$, quelques auteurs ont envisagé un changement de dimensionalité du système, c'est en particulier le cas de Knotek, Pollak, Donovan et Kurtzman [3], qui ont étudié le changement de comportement de films amorphes de $\mathrm{Ge}$ en fonction de l'épaisseur de ceux-ci. Redfield [4] a par ailleurs suggéré que la valeur $x=2$ serait due à une conduction se faisant au sein de chaînes macroscopiques de sites. Il semble toutefois que ce genre d'argument ne puisse guère expliquer la variation de $x$ en fonction de la température.

Pour rendre compte du passage de $x$ de 4 à 2 aux très basses températures Efros et Shklowskii [5] proposent de leur côté un modèle tenant compte de l'interaction Coulombienne entre le site abandonné par l'électron, assimilable à une charge positive dans le milieu, et le site rejoint par cet électron, assimilable à une charge négative. Cette interaction ne peut excéder l'écart énergétique entre les deux niveaux. Cette condition impose à la densité d'états $N(E)$ de s'annuler au niveau de Fermi, et de présenter une forme parabolique autour de $E_{\mathrm{F}}$

$$
N^{\prime}(E)=\frac{K^{3}}{e^{6}}\left(E-E_{\mathrm{F}}\right)^{2},
$$

$K$ étant la constante diélectrique du matériau et $e$ la charge électronique; $N^{\prime}(E)$ doit toujours rester inférieure à $N\left(E_{\mathrm{F}}\right)$, densité d'états dans la bande inter- dite (supposée constante) en l'absence d'interaction Coulombienne. La forme de la densité d'états découlant des hypothèses d'Efros et Shklowskii est illustrée sur la figure $1 b$.

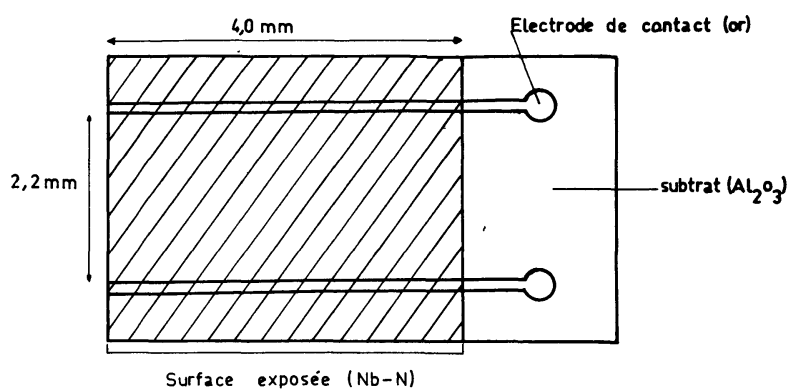

a)

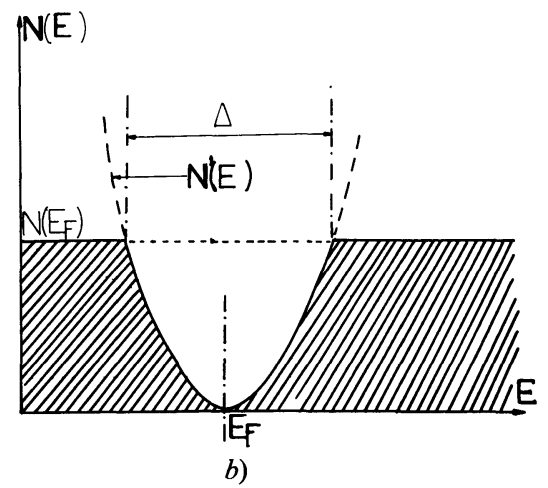

Fig. 1. - a) Représentation schématique des échantillons étudiés ; l'épaisseur est comprise entre 300 et $700 \AA$. b) Allure de la densité d'états dans le gap de mobilité dans la théorie de Efros-Shklowskii.

[a) Diagram of the studied samples; the thickness has a value between 300 and $700 \AA$. b) Typical density of states in the mobility gap in the Efros-Shklowskii's model.]

Appliquant les calculs de Mott en tenant compte de cette forme particulière de la densité d'états, on aboutit à la relation (5) avec $x=2$ et à l'expression suivante de la température $T_{0(2)}$

$$
T_{0(2)}=\frac{e^{2}}{K d k_{\mathrm{B}}} .
$$

Cette théorie prévoit également le passage de la conductivité d'un régime à l'autre; la température $T_{\mathrm{c}}$ de changement entre les comportements exprimés par $x=4$ et $x=2$ dans la relation (5) dépend en particulier de $N\left(E_{\mathrm{F}}\right)$

$$
T_{\mathrm{c}} \simeq \frac{e^{4} \cdot N\left(E_{\mathrm{F}}\right) d}{k_{\mathrm{B}} K^{2}}
$$

Des expériences préliminaires [6] nous avaient conduit à penser que ce modèle de Efros et Shklowskii pouvait expliquer le comportement observé à très basse température. Dans le présent article, à la lumière de résultats expérimentaux plus complets, obtenus sur des alliages $\mathrm{Nb}-\mathrm{N}$, nous montrons que la synthèse des théories de Mott et de Efros-Shklowskii permet de déterminer la densité d'états au niveau de Fermi et conduit à des valeurs acceptables de celle-ci. 
2. Méthodes expérimentales. - Nous avons utilisé pour l'élaboration de nos matériaux la technique classique de pulvérisation cathodique réactive en montage diode-radiofréquence $(13,7 \mathrm{MHz})$, qui a été décrite à plusieurs reprises par différents auteurs. La cathode est constituée de niobium (pureté 99,99\%) et le plasma est formé d'un mélange gazeux (argon/ azote) à la pression totale de $2 \times 10^{-3}$ torr. Les principaux paramètres d'élaboration qui déterminent la structure et les propriétés physiques des couches sont essentiellement la pression partielle de l'azote dans le mélange gazeux et la température $T_{\mathrm{s}}$ du substrat de la couche; cette température est déterminée par circulation, soit d'eau, soit d'un liquide cryogénique. Par ailleurs, le temps de pulvérisation détermine l'épaisseur de nos couches, de 300 à $700 \AA$ pour des durées comprises entre $20 \mathrm{~min}$. et $50 \mathrm{~min}$. La géométrie des échantillons est représentée sur la figure $1 a$; les électrodes de contact, en or, sont déposées par évaporation sous vide, avant la pulvérisation.

Les mesures de la résistance des couches en fonction de la température ont été effectuées en utilisant les procédés classiques en cryogénie : la sonde de température est un capteur au carbone-verre « LakeShore " et la valeur de la résistance est mesurée avec un électromètre « Keithley 616 ».

3. Résultats expérimentaux. - Nous allons maintenant exposer les résultats des mesures de la conductivité en fonction de la température pour des couches minces de nitrure de niobium non métalliques et désordonnées. Nous analyserons ensuite comment on peut interpréter ces résultats à la lumière des théories exposées ci-dessus.

\subsection{VARIATION EXPÉRIMENTALE DE LA RÉSISTANCE} EN FONCTION DE LA TEMPÉRATURE. - La figure 2 représente la variation de la résistance d'un échantillon typique, portée en coordonnées logarithmiques successivement en fonction de $T^{-1 / 2}, T^{-1 / 4}, T^{-1}$ $(2 a, 2 b, 2 c)$.

Dans le domaine des basses températures, la linéarité de $\log R$ en fonction de $T^{-1 / 2}$ est établie; pour des températures plus élevées, le régime, en $T^{-1 / 4}$ est nettement conforme à la loi de Mott, $T_{0(4)}$ étant de l'ordre de $10^{5}$ à $10^{7} \mathrm{~K}$. Ce régime est limité du côté des hautes températures par une température $T_{\mathrm{s}}$ qui peut être inférieure ou supérieure à la température ambiante. Dans le premier cas, il a été possible de mettre en évidence, aux hautes températures, après un important domaine de transition en température, un régime activé en $T^{-1}$ (Fig. $2 c$ ).

Ces trois régimes seront respectivement notés 1 , 2 et 3. La température $T_{\mathrm{c}}$ de changement de régime entre les régimes 1 et 2, ne peut être définie expérimentalement que par un intervalle dont les bornes sont $T_{\mathrm{c} 1}$ et $T_{\mathrm{c} 2}$ (voir Fig. 2) et dont la largeur varie selon les échantillons, de $1 \mathrm{~K}$ à $15 \mathrm{~K}$ environ, et qui situent $T_{\mathrm{c}}$ de $7 \mathrm{~K}$ à $30 \mathrm{~K}$ pour la majorité de nos échantillons.
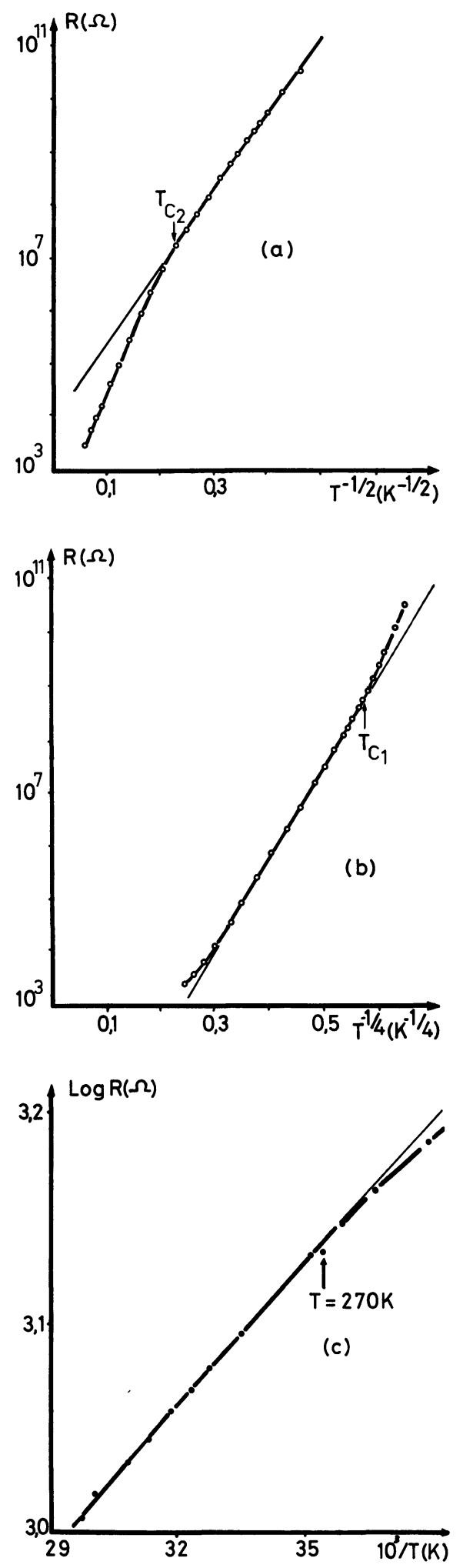

Fig. 2. - Variation de la résistance pour un échantillon typique en fonction de :a) $T^{-1 / 2}$; b) $T^{-1 / 4}$; c) $T^{-1}$. On voit clairement l'existence de trois régimes en fonction de la température. $T_{\mathrm{c} 1}$ et $T_{\mathrm{c} 2}$ représentent les limites expérimentales de la transition entre les régimes en $T^{-1 / 2}$ et $T^{-1 / 4}$.

[Variation of the resistance for a typical sample as a function of : a) $T^{-1 / 2}$; b) $T^{-1 / 4}$; c) $T^{-1}$. The existence of three different regimes clearly appear in the behaviour exhibited by this sample. $T_{\mathrm{c} 1}$ and $T_{\mathrm{c} 2}$ are the experimental limits of the transition between the $T^{-1 / 2}$ and $T^{-1 / 4}$ regimes.] 
Les régimes 2 et 3 sont, lorsqu'ils sont observés tous les deux, séparés par un large domaine en température $\left[T_{\mathrm{A} 1}, T_{\mathrm{A} 2}\right]$. Pour l'un de nos échantillons $T_{\mathrm{A} 1} \simeq 85 \mathrm{~K}$ et $T_{\mathrm{A} 2} \simeq 270 \mathrm{~K}$. Des échantillons, dont la variation de la résistance avec la température est beaucoup moins rapide que pour la moyenne de nos couches, présentent un aspect sensiblement différent (Fig. 3) où l'on n'observe un régime en $T^{-1 / 4}$ que pour une température inférieure à $15 \mathrm{~K}$. Audessus de cette température, contrairement aux autres types d'échantillons, la variation en fonction de la température est plus rapide que pour $T<15 \mathrm{~K}$.

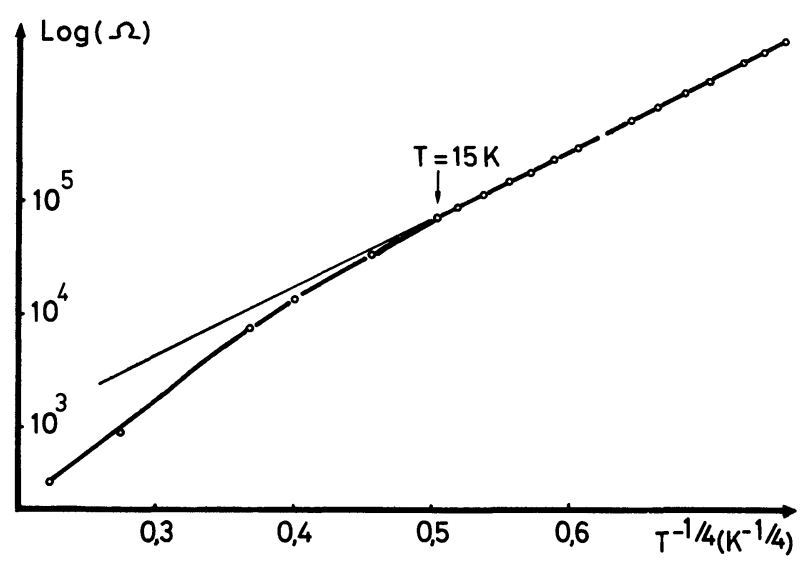

Fig. 3. - Variation de la résistance en fonction de la température pour un échantillon présentant une variation relativement faible.

[Variation of the resistance as a function of the temperature for a sample exhibiting a moderated variation.]
Néanmoins, ce cas qui sera considéré comme issu d'une situation plus complexe par la suite, est momentanément exclu de notre interprétation qui est dans un premier temps réduite aux cas typiques décrits plus haut et illustrés par les figures 2 .

Les régimes 2 et 3 sont interprétés comme traduisant les processus de conduction par sauts entre états localisés respectivement à distance variable et entre proches voisins.

Le régime 1 en $T^{-1 / 2}$ aux basses températures est plus difficile à interpréter du fait de l'absence d'accord des différents auteurs sur cette question. Toutefois, il nous semble intéressant d'analyser les implications des hypothèses d'Efros et Shklowskii relativement à l'existence d'un pseudogap de forme parabolique. La coexistence d'un régime classique de Mott en $T^{-1 / 4}$ et d'un régime en $T^{-1 / 2}$ permet de confronter les expressions de trois températures :

$T_{0(2)}$ et $T_{\mathrm{c}}$ (température de changement de régime) données par la théorie d'Efros et Shklowskii, et $T_{0(4)}$ donnée par la théorie de Mott.

A partir des relations (2), (6) et (7), on trouve aisément que ces trois températures sont reliées par l'expression :

$$
T_{\mathrm{c}} \simeq 18,1 \frac{T_{0(2)}^{2}}{T_{0(4)}}
$$

Le coefficient numérique égal à 18,1 dans l'analyse de Mott, est égal à 16 dans la théorie d'Ambegaokar, Halperin et Langer qui retrouvent, à ce coefficient

Tableau I. - Comparaison entre d'une part la valeur théorique de la température $T_{\mathrm{c}}$ de transition entre les régimes en $T^{-1 / 2}$ et $T^{-1 / 4}$, et d'autre part la plage expérimentale correspondant à cette transition. La deuxième colonne indique le mode de refroidissement du porte-substrat; la troisième colonne donne la valeur du rapport $X_{\mathrm{N}_{2}}=P_{\mathrm{N}_{2}} / P_{\mathrm{tot}}$; de la pression partielle d'azote à la pression totale dans le plasma de pulvérisation au cours de l'élaboration de l'alliage; la quatrième colonne correspond à la durée de la pulvérisation (l'épaisseur de la couche est en gros proportionnelle à cette durée; on obtient environ $300 \AA$ en $20 \mathrm{~min}$.).

[Comparison between the theoretical value of the transition temperature $T_{\mathrm{c}}$ (between the $T^{-1 / 2}$ and the $T^{-1 / 4}$ regimes) and the experimental range of temperatures corresponding to this transition. The second column shows the technique for refrigerating the substrate; the third column gives the value of the ratio, $X_{\mathrm{N}_{2}}=P_{\mathrm{N}_{2}} / P_{\mathrm{tot}}$, of the partial pressure of nitrogen to the total pressure of the plasma during the sputtering process; the fourth column gives the duration of the sputtering (the thickness of the film is approximately proportional to this duration; we typically obtain $300 \AA$ in $20 \mathrm{~min}$.). $]$

\begin{tabular}{|c|c|c|c|c|c|c|c|}
\hline \multirow{2}{*}{$\begin{array}{l}\mathbf{N}^{\circ} \\
\text { éch. }\end{array}$} & \multicolumn{3}{|c|}{ Paramètres d'élaboration } & \multirow[b]{2}{*}{$T_{0(4)}(\mathrm{K})$} & \multirow[b]{2}{*}{$T_{0(2)}(\mathrm{K})$} & \multirow{2}{*}{$\begin{array}{c}\text { Intervalle } \\
\text { expérimental } T_{\mathrm{c}}(\mathrm{K})\end{array}$} & \multirow{2}{*}{$T_{\text {c calculé }}=16 \frac{T_{0(2)}^{2}}{T_{0(4)}}$} \\
\hline & réf. & $X_{\mathrm{N}_{2}}$ & $t$ (min.) & & & & \\
\hline 1 & azote & 0,9 & 40 & $2,8 \times 10^{6}$ & $1,5 \times 10^{3}$ & 14,$7 ; 25,0$ & 13,5 \\
\hline 2 & azote & 1,0 & 30 & $3,5 \times 10^{6}$ & $1,9 \times 10^{3}$ & 15,$0 ; 25,0$ & 16 \\
\hline 3 & eau & 0,8 & 60 & $1,7 \times 10^{6}$ & $2,2 \times 10^{3}$ & $30 ; 60$ & 44 \\
\hline 4 & eau & 1,0 & 60 & $3,6 \times 10^{6}$ & $1,4 \times 10^{3}$ & 7,3 & $8,6^{\circ}$ \\
\hline 5 & sans réf. & 0,8 & 60 & $4,8 \times 10^{6}$ & $1,6 \times 10^{3}$ & 7,7 & 8,8 \\
\hline 6 & sans réf. & 0,9 & 60 & $5,4 \times 10^{6}$ & $1,9 \times 10^{3}$ & 9,4 & 11,1 \\
\hline 7 & sans réf. & 0,8 & 30 & $1,8 \times 10^{5}$ & $4,5 \times 10^{2}$ & $11 ; 20$ & 18,5 \\
\hline
\end{tabular}


près, l'expression de $T_{0(4)}$ de Mott, par un calcul de percolation [2]. La vérification expérimentale de l'expression (9) et donc de la cohérence des théories de Mott et d'Efros-Shklowskii, consiste dans la comparaison entre d'une part la valeur de $T_{\mathrm{c}}$, calculée à partir de cette relation et de valeurs expérimentales de $T_{0(4)}$ et $T_{0(2)}$, et d'autre part l'intervalle expérimental défini plus haut par $T_{\mathrm{c} 1}$ et $T_{\mathrm{c} 2}$.

Le tableau I illustre cette comparaison sur quelques échantillons typiques, pour lesquels les paramètres d'élaboration sont indiqués. Les résultats portés sur ce tableau indiquent un bon accord entre la théorie d'Efros-Shklowskii et l'expérience. Il est à noter que l'expression (7) de $T_{0(2)}$, calculée par Efros et Shklowskii à partir de leur hypothèse de l'existence d'un pseudo-gap parabolique, n'est exacte qu'en ordre de grandeur. A cette réserve près toutefois, nos résultats expérimentaux semblent indiquer que la synthèse des théories de Mott et d'Efros-Shklowskii peut conduire à une représentation réaliste des phénomènes physiques. Nous allons dans la suite apporter des arguments supplémentaires à cette analyse.

3.2 ETUDE DES PARAMÈTRES DE STRUCTURE DU MATÉRIAU. - Dans l'analyse de Mott de la conduction par sauts à distance variable, les expressions (2) et (3) de la température $T_{0(4)}$ et du facteur pré-exponentiel $\sigma_{0(4)}$ font intervenir la densité d'états $N\left(E_{\mathrm{F}}\right)$ au niveau de Fermi, supposée constante dans le gap de mobilité, la longueur de localisation $d$, ainsi que $v_{0}$ définie plus haut comme fréquence liée au spectre de phonons du matériau ; Paul et Mitra [7] ont défini une méthode permettant d'obtenir $N\left(E_{\mathrm{F}}\right)$ et $d$ à partir des résultats expérimentaux. L'expression de $R$ indique que $\sigma_{0(4)}$ n'est pas indépendant de la température; en revanche $\sigma_{0(4)}^{\prime}=\sigma_{0(4)} T^{1 / 2}$ ne dépend pas de $T$. La méthode graphique de ces auteurs consiste à porter

$$
\sigma \sqrt{T}=\sigma_{0(4)}^{\prime} \mathrm{e}^{\left(T / T_{0(4)}\right)^{-1 / 4}}
$$

en coordonnées logarithmiques en fonction de $T^{-1 / 4}$. $\sigma_{0(4)}^{\prime}$ est alors obtenu par extrapolation de la partie rectiligne de la courbe. Les expressions de $N\left(E_{\mathrm{F}}\right)$ et $d$ se déduisent alors des relations (2), (3) et (5) :

$$
\begin{aligned}
N\left(E_{\mathrm{F}}\right) & =\frac{2 \times 10^{48}}{v_{0}^{3}} \sqrt{T_{0(4)} \cdot\left(\sigma_{0(4)}^{\prime}\right)^{3}} \mathrm{~cm}^{-3} \cdot \mathrm{eV}^{-1} \\
d & =\frac{21,2 \times 10^{13}}{v_{0}} \sqrt{\sigma_{0(4)}} \sqrt{\sigma_{0(4)}^{\prime}} \mathrm{cm}^{-1}
\end{aligned}
$$

Nous avons appliqué cette méthode à nos échantillons. La fréquence $v_{0}$, variable suivant les matériaux, se situe vraisemblablement entre $10^{12}$ et $10^{13} \mathrm{~Hz}$; en l'absence de détermination expérimentale, elle a été prise égale à $10^{13} \mathrm{~Hz}$. Pour les deux échantillons concernés pour la figure 4 , nous obtenons ainsi :

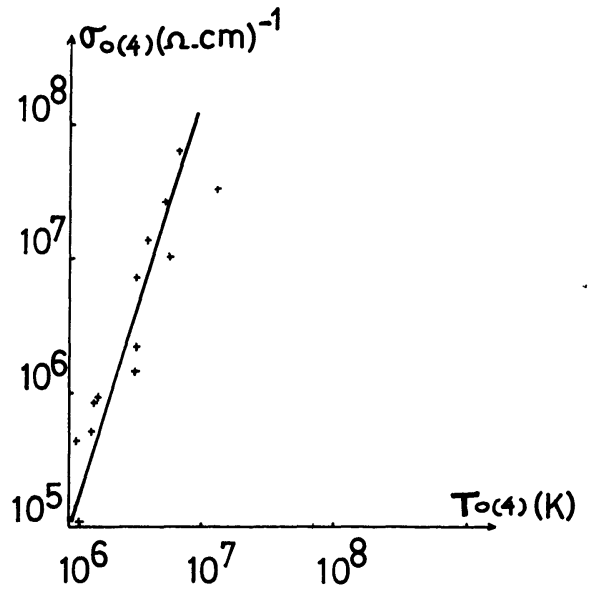

Fig. 4. - Relation entre les coefficients de Mott $\rho_{0(4)}$ et $T_{0(4)}$ pour une série de nos échantillons de $\mathrm{Nb}-\mathrm{N}$. Une certaine corrélation semble ressortir, qui se traduirait par une formule du type

$$
\rho_{0(4)} \propto T_{0(4)}^{-5 / 2} .
$$

[Relation between the two Mott parameters $\rho_{0(4)}$ and $T_{0(4)}$ for numerous Nb-N samples. A 'relation of the type $\rho_{0(4)} \propto T_{0(4)}^{-5 / 2}$ could be inferred from these results and indicates a correlation between the two parameters.]

échantillon a :

$$
N\left(E_{\mathrm{F}}\right)=2,3 \times 10^{38} \mathrm{~cm}^{-3} \cdot \mathrm{eV}^{-1} ; d=5,3 \times 10^{-6} \AA
$$

échantillon $\mathrm{b}$ :

$N\left(E_{\mathrm{F}}\right)=1,5 \times 10^{29} \mathrm{~cm}^{-3} \cdot \mathrm{eV}^{-1} ; \quad d=1,5 \times 10^{-2} \AA$.

De même que les résultats obtenus par Paul et Mitra sur des couches de semi-conducteurs amorphes ( $\mathrm{Si}$, $\mathrm{Ge}, \mathrm{Si}-\mathrm{Ge}$ ), les valeurs trouvées sur nos échantillons sont très différentes des ordres de grandeur physiquement acceptables.

L'accord entre la température expérimentale de changement de régime et celle calculée à partir de la relation (9) semblant relativement satisfaisant compte tenu des réserves liées aux approximations d'Efros et Shklowskii, il nous a paru justifié de poursuivre la synthèse des deux théories, afin de déterminer les valeurs de $N\left(E_{\mathrm{F}}\right)$ et de $d$.

Les expressions de $T_{0(2)}$ et de $T_{0(4)}$ permettent de déterminer $N\left(E_{\mathrm{F}}\right)$ et $d$, moyennant seulement une estimation de la constante diélectrique $K$, on obtient

$$
\begin{aligned}
d & =\frac{e^{2}}{k_{\mathrm{B}} T_{0(2)}} \frac{1}{K} \\
N\left(E_{\mathrm{F}}\right) & \simeq \frac{16}{e^{6}} \frac{k_{\mathrm{B}}^{2} T_{0(2)}^{3}}{T_{0(4)}} K^{3} .
\end{aligned}
$$

A partir de cette méthode, d'autres grandeurs peuvent être atteintes à partir des valeurs expérimentales de $T_{0(2)}$ et $T_{0(4)}$ :

- la largeur du pseudo-gap Coulombien dont l'expression est

$\Delta \simeq 2 \frac{e^{3}}{K^{3 / 2}} N\left(E_{\mathrm{F}}\right)^{1 / 2} \quad$ d'après Efros et Shklowskii, 
Tableau II. - Détermination des paramètres physiques $N\left(E_{\mathrm{F}}\right)$ et $d$ (distance de localisation) soit à partir de la méthode proposée par Paul et Mitra, soit en utilisant notre synthèse des théories de Mott et de Efros-Shklowskii (nous avons pris la constante diélectrique égale à 10). Les quatre premières colonnes ont la même signification que dans le tableau $I$; les valeurs de $T_{0(4)}$ et $T_{0(2)}$ dans les colonnes 5 et 6 correspondent à des déterminations expérimentales; les deux dernières colonnes donnent la valeur de la distance la plus probable de saut à deux températures $(20 \mathrm{~K}$ et $100 \mathrm{~K})$.

[Determination of the physical parameters $N\left(E_{\mathrm{F}}\right)$ and $d$ (length of localization) by using either Paul and Mitra's method, or our synthesis of the theories of Mott and Efros-Shklowskii (the dielectric constant has been taken to be 10). The first four columns have the same meaning as in table $I$; the values of $T_{0(4)}$ and $T_{0(2)}$ in columns 5 and 6 are experimental values; the last two columns give the value of the most probable hopping length at two temperatures $(20 \mathrm{~K}$ and $100 \mathrm{~K})$.]

\begin{tabular}{|c|c|c|c|c|c|c|c|c|c|c|c|c|c|}
\hline \multirow[b]{2}{*}{$\begin{array}{r}\mathbf{N}^{\circ} \\
\text { éch }\end{array}$} & \multicolumn{3}{|c|}{$\begin{array}{c}\text { Conditions de } \\
\text { préparation }\end{array}$} & \multirow[b]{2}{*}{$\begin{array}{l}T_{0(4)} \\
(\mathrm{K})\end{array}$} & \multirow[b]{2}{*}{$\begin{array}{l}T_{0(2)} \\
(\mathrm{K})\end{array}$} & \multicolumn{3}{|c|}{ Méthode Paul et Mitra } & \multicolumn{5}{|c|}{ Méthode utilisant la théorie de Efros-Shklowskii } \\
\hline & $\begin{array}{l}\text { type de } \\
\text { refroid. }\end{array}$ & $X_{\mathrm{N}_{2}}$ & $\begin{array}{l}\text { durée } \\
\text { pulv. } \\
\text { (min.) }\end{array}$ & & & $\begin{array}{l}T_{0(4)} \\
(\mathrm{K})\end{array}$ & $\begin{array}{c}N\left(E_{\mathrm{F}}\right) \\
\left(\mathrm{cm}^{-3} \cdot \mathrm{eV}^{-1}\right)\end{array}$ & $\begin{array}{c}d \\
(\AA)\end{array}$ & $\begin{array}{c}N\left(E_{\mathrm{F}}\right) \\
\left(\mathrm{cm}^{-3} \cdot \mathrm{eV}^{-1}\right)\end{array}$ & $\begin{array}{c}d \\
(\AA)\end{array}$ & $\begin{array}{c}\Delta \\
(\mathrm{eV})\end{array}$ & $\begin{array}{c}R_{T=20 \mathrm{~K}} \\
(\AA)\end{array}$ & $\begin{array}{c}R_{T}=100 \mathrm{~K} \\
(\AA)\end{array}$ \\
\hline 1 & sans réf. & 0,8 & 60 & $4,8 \times 10^{6}$ & $1,6 \times 10^{3}$ & $3,6 \times 10^{10}$ & $2,3 \times 10^{38}$ & $5,3 \times 10^{-6}$ & $3,5 \times 10^{19}$ & 10 & $2,5 \times 10^{-2}$ & 81 & 54 \\
\hline 2 & sans réf. & 0,8 & 30 & $1,8 \times 10^{5}$ & $4,5 \times 10^{2}$ & $5,0 \times 10^{7}$ & $1,5 \times 10^{29}$ & $1,5 \times 10^{-2}$ & $2,1 \times 10^{19}$ & 38 & $1,6 \times 10^{-2} \mid$ & 140 & 92 \\
\hline 3 & $\begin{array}{l}\text { refroid. } \\
\text { eau }\end{array}$ & 1,0 & 60 & $3,6 \times 10^{6}$ & $1,4 \times 10^{3}$ & $7,9 \times 10^{6}$ & $2,4 \times 10^{27}$ & $2,5 \times 10^{-2}$ & $3,0 \times 10^{19}$ & 12 & $1,9 \times 10^{-2}$ & 96 & 64 \\
\hline 4 & sans réf. & 0,9 & 20 & $9,9 \times 10^{6}$ & $5,1 \times 10^{3}$ & $3,9 \times 10^{10}$ & $4,4 \times 10^{38}$ & $3,3 \times 10^{-4}$ & $5,2 \times 10^{20}$ & 3 & $8,0 \times 10^{-2}$ & 34 & 22 \\
\hline 5 & réf. azote & 1,0 & 60 & $2,1 \times 10^{6}$ & $1,5 \times 10^{3}$ & $3,5 \times 10^{9}$ & $4,5 \times 10^{34}$ & $7 \times 10^{-5}$ & $6,5 \times 10^{19}$ & 11 & $2,8 \times 10^{-2}$ & 77 & 51 \\
\hline
\end{tabular}

- la distance de saut la plus probable $R$ à une température $T$ donnée par

$$
R=\left(\frac{9}{8 \pi}\right)^{1 / 4} \frac{e^{2} T_{0(4)}^{1 / 4}}{2 K k_{\mathrm{B}} T_{0(2)}} T^{-1 / 4}
$$

La constante diélectrique $K$ varie relativement peu d'un matériau à un autre. Dans notre cas, en l'estimant comprise entre 5 et 15 soit $K=10$ avec une incertitude relative égale à $1 / 2$, on voit que l'incertitude absolue qui en résulte sur $N\left(E_{\mathrm{F}}\right)$ reste inférieure à un ordre de grandeur.

Le tableau II indique ces différentes valeurs pour 5 échantillons définis par leurs paramètres d'élaboration, ainsi que les valeurs obtenues par la méthode de Paul et Mitra décrite ci-dessus. Tandis que les valeurs de la densité d'états $N\left(E_{\mathrm{F}}\right)$ issues de cette dernière méthode s'avèrent très dispersées (entre $10^{30}$ et $10^{27} \mathrm{~cm}^{-3} \cdot \mathrm{eV}^{-1}$ ) et beaucoup trop élevées, celles issues de notre analyse sont relativement groupées $10^{19}-10^{20} \mathrm{~cm}^{-3} \cdot \mathrm{eV}^{-1}$, et présentent un ordre de grandeur convenable. De plus la valeur de la longueur de localisation des états localisés, quelques paramètres cristallins, est raisonnable; il en est de même pour la largeur $\Delta$, de l'ordre de $10^{-2} \mathrm{eV}$. Pour l'échantillon concerné par la figure $2 c$, la valeur de $\Delta$ égale à $1,9 \times 10^{-2} \mathrm{eV}$ peut être comparée à l'écart énergétique $(\Delta E)_{0}$ entre proches voisins calculé à partir de la pente de la droite représentant $-\log \sigma$ en fonction de $T^{-1}$, trouvé égal à

$$
4,5 \times 10^{-2} \mathrm{eV} \text {. }
$$

Il est satisfaisant de constater que $\Delta>(\Delta E)_{0}$ puisque le régime de Mott en $T^{-1 / 4}$ a été effectivement observé.
L'échec de la méthode de Paul et Mitra qui ne donne pas des ordres de grandeur raisonnables pour $N\left(E_{\mathrm{F}}\right)$ et $d$ est vraisemblablement imputable à la forme du facteur pré-exponentiel utilisée par ces auteurs; il semble que la réalité soit plus complexe que celle exprimée par la relation (3). En particulier Wuertz et Thomas [8] ont constaté que le facteur pré-exponentiel est très sensible aux conditions de préparation et peut varier de 7 ordres de grandeur sur du silicium ou du germanium amorphe; ils attribuent plus particulièrement à la fréquence $v_{0}$, cette sensibilité de $\sigma_{0(4)}$ aux conditions de préparation. Wuertz et Thomas n'établissent cependant pas une expression de $\sigma_{0}$, mais calculent qu'il est proportionnel à $T^{-\gamma}, \gamma$ pouvant varier de $1 / 2$ (comme dans la relation de Mott) à $-5 / 2$ en fonction des conditions de préparation. Par ailleurs, ces auteurs font également dépendre le facteur pré-exponentiel du paramètre $T_{0(4)}$, par une relation du type

$$
\rho_{0(4)} \propto\left(\frac{T_{0(4)}}{T}\right)^{-\gamma} .
$$

Nous avons porté sur la figure 4 les points représentatifs de chacun de nos échantillons, dans un graphe de $-\log \sigma_{0(4)}$ en fonction de $\log T_{0(4)}$. Ces points se situent aux environs d'une droite dont la pente est proche de $-5 / 2$, ce qui pourrait confirmer la relation de Wuertz et Thomas, si on admet que dans notre mode de fabrication, le facteur $\gamma$ reste sensiblement le même pour l'ensemble des échantillons.

Il résulte en tout cas de ces observations que la forme du facteur pré-exponentiel $\sigma_{0(4)}$ est beaucoup plus complexe que celle trouvée par Mott et sur laquelle 
s'appuient Paul et Mitra. Cette réserve ne remet toutefois pas en cause les principes physiques de saut entre états localisés proches voisins et à distance variable, qui n'imposent que la forme du facteur exponentiel lui-même. C'est cette circonstance qui peut précisément expliquer que Paul et Mitra trouvent un ordre de grandeur convenable pour le rapport $R / d$ à température donnée.

En effet, ce rapport a pour expression, dans l'analyse de Mott :

$$
\frac{R}{d}=\frac{9}{8}\left(\frac{T^{-1}}{k_{\mathrm{B}} d^{3} N\left(E_{\mathrm{F}}\right)}\right)^{1 / 4}\left(\frac{T}{T_{0(4)}}\right)^{-1 / 4}
$$

il ne dépend donc pas de $\sigma_{0(4)}$.

4. Conclusion. - Les résultats obtenus sur l'alliage $\mathrm{NbN}$, que nous venons d'exposer, mettent en évidence la possibilité de la coexistence de plusieurs modes de conduction par sauts entre états localisés dans un même matériau en fonction de la gamme de températures considérée. A ce titre, ils paraissent donc présenter un double intérêt :

a) Ils apportent un support expérimental au modèle de Efros et Shklowski, qui fait intervenir à très basse température les corrélations électroniques dans le processus de saut à distance variable. Il est d'ailleurs possible que nos expériences confirment plus ce phénomène général plutôt que le modèle particulier des auteurs en question.

b) Ils indiquent un moyen expérimental d'obtenir les valeurs des paramètres microscopiques caractérisant le matériau étudié, dans la mesure, où celui-ci présente effectivement les deux régimes de conduction à basse température.

\section{Bibliographie}

[1] Mott, N. F., Adv. Phys. 16 (1967) 49 ; Philos. Mag. 19 (1969) 835 ; J. Phys. C 8 (1975) 239; Philos. Mag. 34 (1976) 643.

[2] Ambegaokar, V., Halperin, B. I. and Langer, J. S., Phys. Rev. B 4 (1971) 2612.

[3] Knotek, M. L. and Pollak, M., Donovan, T. M. and KurtzMAN, H., Phys. Rev. Lett. 30 (1973) 853.
[4] Redfield, D., Phys. Rev. Lett. 30 (1973) 1319; Adv. Phys. 24 (1975) 463.

[5] Efros, A. L. and Shklowskit, B. I., J. Phys. C 8 (1975) 49.

[6] Baixeras, J., Chevalier, J. and ANDro, P., Philos. Mag. 36 (1977) 1269.

[7] Paul, D. K., Mrtra, S. S., Phys. Rev. Lett. 31 (1973) 1000

[8] Wuertz, D., Thomas, P., Phys. Status Solidi (b) 88 (1978) K 73. 